TECHNICAL COMMENT

PLANT ECOLOGY

\section{Comment on "Worldwide evidence of a unimodal relationship between productivity and plant species richness"}

\author{
Andrew T. Tredennick, ${ }^{1 *} \dagger$ Peter B. Adler, ${ }^{1} \dagger$ James B. Grace, ${ }^{2} \dagger$ W. Stanley Harpole, ${ }^{3} \dagger$ \\ Elizabeth T. Borer, ${ }^{4} \dagger$ Eric W. Seabloom, ${ }^{4} \dagger$ T. Michael Anderson, ${ }^{5}$ Jonathan D. Bakker, ${ }^{6}$ \\ Lori A. Biederman, ${ }^{7}$ Cynthia S. Brown, ${ }^{8}$ Yvonne M. Buckley, ${ }^{9}$ Chengjin Chu, ${ }^{10}$ Scott L. Collins, ${ }^{11}$ \\ Michael J. Crawley, ${ }^{12}$ Philip A. Fay, ${ }^{13}$ Jennifer Firn, ${ }^{14}$ Daniel S. Gruner, ${ }^{15}$ Nicole Hagenah, ${ }^{16}$ \\ Yann Hautier, ${ }^{17}$ Andy Hector, ${ }^{18}$ Helmut Hillebrand, ${ }^{19}$ Kevin Kirkman, ${ }^{16}$ \\ Johannes M. H. Knops, ${ }^{20}$ Ramesh Laungani, ${ }^{21}$ Eric M. Lind, ${ }^{4}$ Andrew S. MacDougall, ${ }^{22}$ \\ Rebecca L. McCulley, ${ }^{23}$ Charles E. Mitchell, ${ }^{24}$ Joslin L Moore, ${ }^{25}$ John W. Morgan, ${ }^{26}$ \\ John L. Orrock, ${ }^{27}$ Pablo L. Peri, ${ }^{28}$ Suzanne M. Prober, ${ }^{29}$ Anita C. Risch, ${ }^{30}$ \\ Martin Schütz, ${ }^{30}$ Karina L. Speziale, ${ }^{31}$ Rachel J. Standish, ${ }^{32}$ Lauren L. Sullivan, ${ }^{4}$ \\ Glenda M. Wardle, ${ }^{33}$ Ryan J. Williams, ${ }^{34}$ Louie H. Yang ${ }^{35}$
}

Fraser et al. (Reports, 17 July 2015, p. 302) report a unimodal relationship between productivity and species richness at regional and global scales, which they contrast with the results of Adler et al. (Reports, 23 September 2011, p. 1750). However, both data sets, when analyzed correctly, show clearly and consistently that productivity is a poor predictor of local species richness.

F raser et al. (1) collected a worldwide data set to examine the relationship between productivity and species richness at global and local scales. They present their results as a direct contrast with the results of Adler et al. (2). However, their presentation obscures substantial areas of agreement, and where results between the two studies do differ, problems in Fraser et al.'s statistical analysis amplify the apparent differences.

The most important area of agreement is the low explanatory power of the "humped-back model" (HBM), in which species richness peaks at intermediate productivity and declines at low and high productivity. Fraser et al. fit a bivariate relationship between productivity and diversity that accounts for less than $1 \%$ of the observed variation in species richness in their data (Table 1 , marginal $R^{2} \mathrm{~s}$ for the Fraser et al. data set). The same is true for an analysis of the Adler et al. data set using a generalized linear mixed model (GLMM) with a block nested within-site randomeffects structure (Table 1, marginal $R^{2}$ s for the Adler et al. data set). Thus, the analyses in both Adler et al. and Fraser et al. demonstrate that productivity is an uninformative predictor of richness for most grasslands. A combined analysis using both data sets yields similar results (Table 1).

A second point of agreement is the difficulty of inferring process from bivariate patterns. The HBM can arise through a wide array of mechanisms $(3,4)$, meaning that the detection of a unimodal pattern does not provide evidence for any particular mechanism.
Adler et al. argued, "[e]cologists should focus on fresh, mechanistic approaches to understanding the multivariate links between productivity and richness" (2). Fraser et al. also concluded "more work is needed to determine the underlying causal mechanisms that drive the unimodal pattern" and called for "additional efforts to understand the multivariate drivers of species richness."

The key disagreement between Fraser $e t$ al. and Adler et al. concerns the statistical significance of the quadratic term that determines the downward concavity of the richness productivity relationship. Adler et al. found little evidence for a concave-down relationship at the site scale ( $2 \%$ of 48 sites) [figure 2 in (2)] and at the global scale reported a significant effect but noted that it was sensitive to choices about which sites to include in the analysis [figure 3 in (2)]. In contrast, Fraser et al. found that $68 \%$ of 28 site-level relationships were significantly concave-down [figure 2A in (I)], and in a global extent regression, across all sites, the negative quadratic term their analysis at the site level is flawed, and the presentation of the global regression in their main figure is misleading.

The site-level regressions reported by Fraser et al. and displayed in their figure $2 \mathrm{~A}$ do not include the proper random-effects structure. An important feature of the Fraser et al. design was explicitly selecting areas (i.e., grids) to sample Adler $e t$ al. located blocks of plots randomly with respect to local productivity gradients. To properly had a significant, and robust, $P$ value. However, across productivity gradients within sites, whereas reflect their sampling design, in which each "grid" of quadrats was located at one point along the within-site productivity gradient, each site-level

Department of Wildland Resources and the Ecology Center, Utah State University, 5230 Old Main, Logan, UT 84322. USA. ${ }^{2}$ U.S. Geological Survey, Wetland and Aquatic Research Center, 700 Cajundome Boulevard, Lafayette, LA 70506, USA. ${ }^{3}$ Department of Physiological Diversity, Helmholtz Center for Environmental Research - UFZ, Permoserstrasse 15, 04318 Leipzig, Germany. ${ }^{4}$ Department of Ecology, Evolution, and Behavior, University of Minnesota, St. Paul, MN 55108, USA. ${ }^{5}$ Department of Biology, Wake Forest University, Box 7325 Reynolda Station, Winston-Salem, NC 27109, USA. ${ }^{6}$ School of Environmental and Forest Sciences, University of Washington, 3501 NE 41st Street, Box 354115, Seattle, WA 98195, USA ${ }^{7}$ Ecology, Evolution and Organismal Biology, lowa State University, 251 Bessey Hall, Ames, IA 50010, USA ${ }^{8}$ Department of Bioagricultural Sciences and Pest Management, Colorado State University, 307 University Avenue, Fort Collins, CO 80523, USA. ${ }^{9}$ School of Natural Sciences, Trinity College Dublin, University of Dublin, Zoology, Dublin 2, Ireland. ${ }^{10}$ School of Life Sciences, Sun Yat-sen University, Xingang Xi Road 135, Guangzhou, 510275, China. ${ }^{11}$ Department of Biology, University of New Mexico, Albuquerque, NM 87131, USA. ${ }^{12}$ Department of Biology, Imperial College London, Silwood Park, Ascot, SL5 7PY, UK. ${ }^{13}$ Grassland, Soil, and Water Research Laboratory, USDA-ARS, 808 East Blackland Road, Temple, TX 76502, USA. ${ }^{14}$ School of Earth, Environmental and Biological 42 Sciences, Queensland University of Technology (QUT), Gardens Point, Brisbane, Queensland, Australia, 4001. ${ }^{15}$ Department of Entomology, University of Maryland, 4112 Plant Sciences, College Park, MD 20742, USA. ${ }^{16}$ School of Life Sciences, University of KwaZulu-Natal, 1 Carbis Road, Pietermaritzburg, 3201, South Africa. ${ }^{17}$ Department of Biology, Ecology and Biodiversity group, Utrecht University, Padualaan 8, $3584 \mathrm{CH}$ Utrecht, Netherlands. ${ }^{18}$ Department of Plant Sciences, University of Oxford, South Parks Road, Oxford, OX1 3RB, UK. ${ }^{19}$ Institute for Chemistry and Biology of the Marine Environment, Carl-von-Ossietzky University Oldenburg, Schleusenstrasse 1, 26382 Wihlhemshaven, Germany. ${ }^{20}$ School of Biological Sciences, University of Nebraska, 211 Manter Hall, Lincoln, NE 68588, USA. ${ }^{21}$ Biology Department, Doane College, 1014 Boswell Avenue, Crete, NE 68333, USA. ${ }^{22}$ Department of Integrative Biology, University of Guelph, 50 Stone Road, Guelph, Ontario, Canada N1G 2W1. ${ }^{23}$ Department of Plant and Soil Science, University of Kentucky, N-222D Ag Science North, Lexington, KY 40546-0091, USA. ${ }^{24}$ Department of Biology, University of North Carolina at Chapel Hill, CB\#3280, Chapel Hill, NC 27599, USA. ${ }^{25}$ School of Biological Sciences, Monash University, Clayton Campus, Wellington Road, Clayton 3800 Victoria, Australia. ${ }^{26}$ Department of Ecology, Environment and Evolution, La Trobe University, Kingsbury Drive, Bundoora 3086, Victoria, Australia. ${ }^{27}$ Department of Zoology, University of Wisconsin, 430 Lincoln Drive, Madison, WI 53706, USA. ${ }^{28}$ Department of Forestry, Agriculture and Water, Southern Patagonia National University-INTA-CONICET, CC 332 (CP 9400), Río Gallegos, Santa Cruz, Patagonia, Argentina. ${ }^{29}$ Commonwealth Scientific and Industrial Research Organisation Land and Water, Private Bag 5 , Wembley, WA 6913, Australia. ${ }^{30}$ Community Ecology, Swiss Federal Institute for Forest, Snow and Landscape Research, Zuercherstrasse 111, 8903 Birmensdorf, Switzerland. ${ }^{31}$ Department of Ecology, INIBIOMA (CONICET-UNCO), Quintral 1250, Bariloche (8400), Rio Negro, Argentina. ${ }^{32}$ School of Veterinary and Life Sciences, Murdoch University, Perth, Western Australia, 90 South Street, Murdoch, Western Australia $6150 .{ }^{33}$ School of Biological Sciences, University of Sydney, Heydon-Laurence Building, A08, University of Sydney, Sydney, NSW, 2006, Australia. ${ }^{34}$ Agricultural and Biosystems Engineering, lowa State University, Ames, IA, USA. ${ }^{35}$ Department of Entomology and Nematology, University of California, Davis, One Shields Avenue, Davis, CA 95616, USA.

*Corresponding author. E-mail: atredenn@gmail.com †Core authors that led the analysis and wrote the paper. All other authors, listed alphabetically, are Nutrient Network members and/or coauthors of Adler et al. (2011) who sign our Comment in support to show consensus among the Nutrient Network. 
Table 1. Results from global-extent GLMMs for both data sets. Results from regressions with and without a quadratic effect of productivity on species richness across all sites. Both models include a random-effects structure of grid nested within site (Fraser et al.) or block nested within site (Adler et al.). Marginal and conditional $R^{2}$ values estimated using $(7,8)$. For the combined analysis, we use the same grid (or block) nested within-site random-effects structure and also include a "study" random effect.

\begin{tabular}{|c|c|c|c|c|}
\hline Data set & Model type & $\begin{array}{c}\text { Marginal } R^{2} \\
\text { (variance explained by } \\
\text { fixed effects) }\end{array}$ & $\begin{array}{c}\text { Conditional } R^{2} \\
\text { (variance explained by } \\
\text { fixed + random effects) }\end{array}$ & $\begin{array}{l}\text { Root mean square } \\
\text { error (in units of } \\
\text { species number) }\end{array}$ \\
\hline Fraser et al. & Linear & 0.00007 & 0.84 & 8.5 \\
\hline Adler et al. & Linear & 0.0007 & 0.79 & 7.7 \\
\hline Adler et al. & Quadratic & 0.001 & 0.78 & 7.7 \\
\hline Combined & Linear & 0.00005 & 0.82 & 8.4 \\
\hline
\end{tabular}

Fig. 1. Species richness as a function of biomass production at the site level (colored lines) and at the global extent (heavy black line). These regressions are the same as presented by Fraser et al. except that we included a grid random effect for the site-level regressions, and we show the proper global extent regression line from a GLMM with grid nested within site. Nonsignificant regression fits are not plotted.

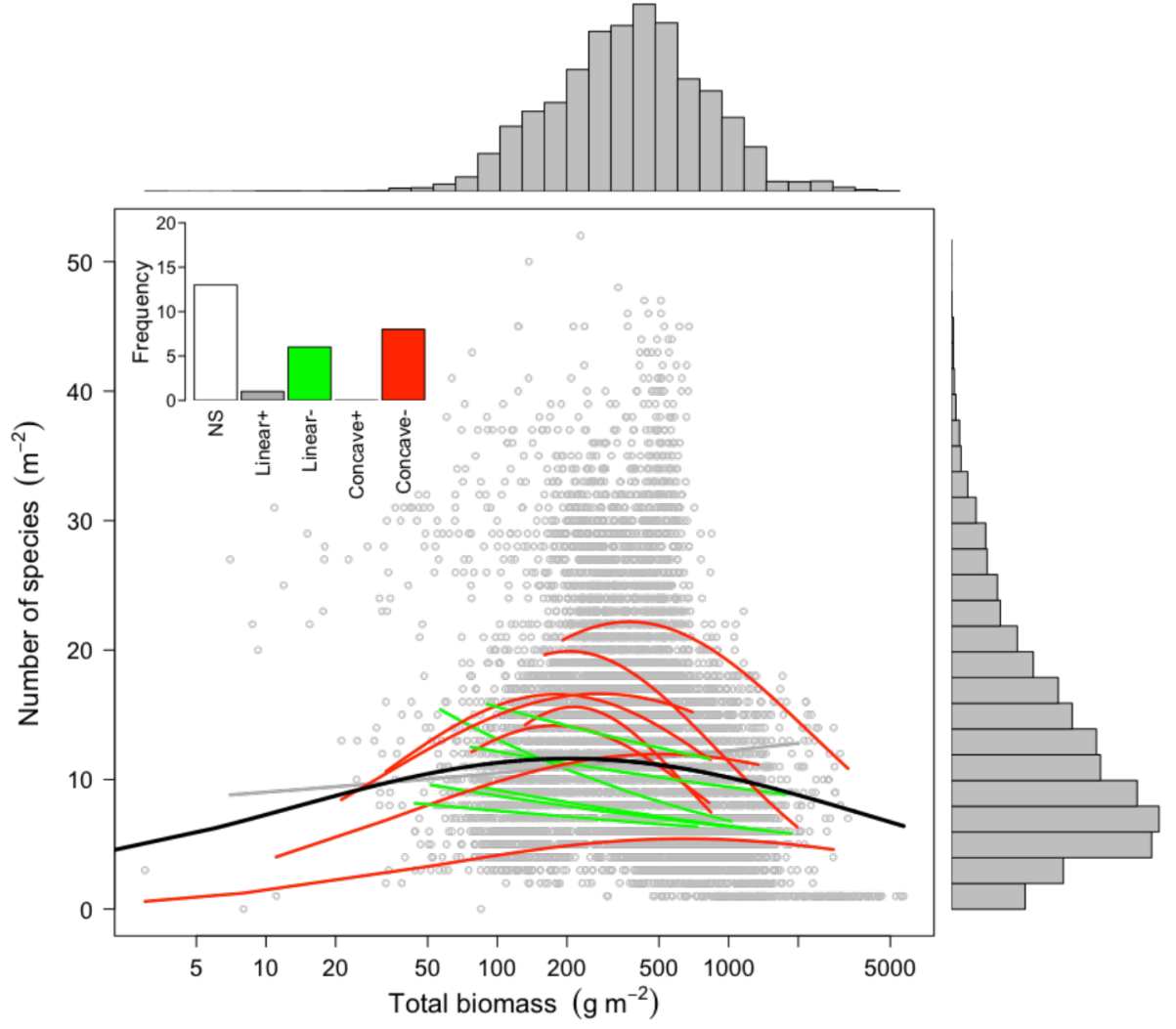

nificant $(P<0.0001)$, the valid relationship is much weaker than the relationship presented by Fraser et al. (Fig. 1, heavy black line, and Table 1).

Despite Fraser et al.'s assertion that their results are diametrically opposed to those presented in Adler et al., the degree of concordance is impressive. In both data sets, the variance explained by the addition of a quadratic term is virtually indistinguishable from that of a linear model (Table 1). In fact, in both data sets the random effects of site and grid (block for Adler et al.) explain much more of the variation in species richness than productivity, the supposed mechanistic driver of species richness (Table 1). Further- more, with the appropriate statistical treatment, the main difference in our results-the strength of evidence for a significant quadratic term-appears smaller.

A continued focus on this bivariate relationship hinders progress toward understanding the underlying multivariate causal relationship (4) and the development of truly predictive models. It is time to focus on effect sizes and variance explained rather than just $P$ values. The title of Adler et al.'s paper, "Productivity is a poor predictor of plant species richness," would be a perfectly appropriate title for the Fraser $e t a l$. paper, too. 


\section{REFERENCES AND NOTES}

1. L. H. Fraser et al., Science 349, 302-305 (2015).

2. P. B. Adler et al., Science 333, 1750-1753 (2011).

3. J. B. Grace et al., Science 335, 6075 (2012).

4. J. B. Grace et al., Nature 529, 10.1038/nature16524 (2016).

5. We used the "Ime4" package in the statistical programming environment R to fit the GLMMs at the site and global extents. Some models struggled to converge on coefficient estimates, a well-known issue with mixed-effects models. We conducted the analyses using different optimizers to make sure that our results are robust (they are), and we did our own checks of model diagnostics to make sure that the warnings could be ignored (they could). Lastly, we fit a hierarchical mixed-effects model using a Bayesian approach to make sure we obtained consistent results (we did). All of our analyses and results can be found on GitHub at http://github.com/atredennick/prodDiv and as release v0.1, https://github.com/atredennick/prodDiv/tag/v0.1

6 . There are four sites, out of 28 , that have only two grids. In only one case did this result in inadequate fits of the GLMM model with a "grid" random effect. We therefore fit that one site with a generalized linear model with no random effects.

7. S. Nakagawa, H. Schielzeth, Methods Ecol. Evol. 4, 133-142 (2013). 8. J. Lefcheck, R-squared for generalized linear mixed-effects models (2014); https://github.com/jslefche/rsquared.glmm

\section{ACKNOWLEDGMENTS}

We thank L. Fraser and colleagues for making their analyses and data openly available. D. Johnson, USGS, provided comments on an earlier version of the manuscript. J.B.G. was supported by the USGS Ecosystems and Climate and Land Use Change Programs. The use of trade, firm, or product names is for descriptive purposes only and does not imply endorsement by the U.S. Government. USDA is an equal opportunity employer. We also acknowledge support from the National Science Foundation Research Coordination Network (NSF-DEB-1042132) and Long Term Ecological Research (NSF-DEB-1234162 to Cedar Creek LTER) programs, and the Institute on the Environment (DG-0001-13).

16 October 2015; accepted 17 December 2015 10.1126/science.aad6236 